\title{
Incidental changes in orthographic processing in the native language as a function of learning a new language late in life
}

(1)

Maria Borragan; Aina Casaponsa²; Eneko Antón; Jon Andoni Duñabeitia ${ }^{4,5 *}$

${ }^{1}$ BCBL, Basque Center on Cognition, Brain and Language; San Sebastian, Spain

2 Deparment of Linguisitcs and English Language, Lancaster University; Lancaster, England

3 Humanitate eta Hezkuntza Zientzien fakultatea, Mondragon Unibertsitatea; Mondragon, Spain

${ }^{4}$ Centro de Ciencia Cognitiva (C3), Universidad Nebrija; Madrid, Spain

5 Department of Languages and Culture, Faculty of Humanities, Social Sciences, and Education, The Arctic University of Norway; Troms $\varnothing$, Norway.

\section{Abstract}

Acquiring a second alphabetic language also entails learning a new set of orthographic rules and specific patterns of grapheme combinations (namely, the orthotactics). The present longitudinal study aims to investigate whether orthotactic sensitivity changes over the course of a second language learning program. To this end, a group of Spanish monolingual old adults completed a Basque language learning course. They were tested in different moments with a language decision task that included pseudowords that could be Basque-marked, Spanish-marked or neutral. Results showed that the markedness effect varied as a function of second language acquisition, showing that learning a second language changes the sensitivity not only to the orthographic patterns of the newly acquired language, but to those of the native language too. These results demonstrate that the orthographic representations of the native language are not static and that experience with a second language boosts markedness perception in the first language.

*Contact information: jdunabeitia@nebrija.es

\section{Keywords}

Orthotactics; orthographic regularities; markedness; second language learning. 


\section{Introduction}

Learning a new language not only involves acquiring new vocabulary, grammar, phonology and syntactic rules, but also acquiring the implicit statistical probabilities regarding the new language's orthographic structure, such as orthotactics. Orthotactics are the patterns of grapheme combinations in written words (see Conway, Bauernschmidt, Huang, \& Pisoni, 2010; Krogh, Vlach, \& Johnson, 2013), and they are learned implicitly by extracting the sublexical regularities of words. People become sensitive to these regularities even after little exposure to printed words (Chetail \& Content, 2017), developing a high sensitivity to letter sequences belonging to one's language (Miller, Bruner, \& Postman, 1954; Owsowitz, 1963).

When readers are exposed to one or several languages, they pick up statistical orthotactic regularities in an unconscious manner, and these seemingly automatically extracted patterns guide ulterior language processing. For instance, a Spanish-English bilingual can easily detect that the word txerri (the Basque word for pig) is neither an English nor a Spanish word solely on the basis of the statistical orthotactic regularities of its constituents, since the bigram tx is not present in the English or Spanish vocabulary. Hence, native speakers of English or Spanish do not need to know the meaning of txerri, or have any knowledge of Basque, in order to decide that this word does not belong to their native languages. When we learn a second language (L2) with an alphabet that maps onto our native one, a similar process of extracting statistical orthotactic regularities takes place (Bordag, Kirschenbaum, Rogahn, \& Tschirner, 2017; Comesaña, Soares, Sánchez-Casas, \& Lima, 2012). Thus, it seems plausible that as we become more proficient in a second language, the new statistical regularities would be better integrated within the preexisting set, leading to a change in our sensitivity to them. In other words, it seems reasonable to predict that the general sensitivity to the orthotactics of both first and second language would change once the new regularities have entered into the system. With this in mind, this study aims to investigate how learning a second language could change the sensitivity to statistical orthotactic regularities from both languages. 

orthographic markedness (namely, the use of language-specific letter combinations) has demonstrated that young bilingual adults, as well as young monolingual adults, are highly sensitive to violations of the statistical orthotactic regularities of the native language (Casaponsa, Carreiras, \& Duñabeitia, 2014; Vaid \& Frenck-Mestre, 2002). In the study by Casaponsa et al. (2014), Spanish monolinguals and Spanish-Basque bilinguals performed a language decision task on Spanish and Basque words. Critically, some of the Basque words included highly distinctive marked bigrams, while others did not. All groups were faster at detecting letter strings that violated Spanish orthotactics as compared to other strings, showing a recognition advantage for Basque words with marked bigrams (e.g., etxe, the Basque word for house, which contains the bigram ' $t x$ ' that does not exist in Spanish). These results showed that even monolinguals can easily detect letter patterns that do not align with their previous implicit orthographic knowledge. Importantly, this suggests that people develop a certain degree of sensitivity to letter sequences that do not conform to their native orthotactic rules, regardless of whether they know the language of the words or not. occurs at an early, semantics-free stage of visual word recognition. Consequently, language attribution mechanisms triggered by orthotactic patterns appear to take place at a sub-lexical level, before access to lexical and semantic representations (see BIA+ extended model, Van Kesteren, Dijkstra, \& de Smedt, 2012; see also BIA+S model, Casaponsa, Thierry, \& Duñabeitia, 2020). Studies exploring the influence of sub-lexical orthographic cues on event-related potential (ERP) patterns related to automatic and unconscious processing of language switches corroborate this idea (e.g., Casaponsa, Carreiras, \& Duñabeitia, 2015; Casaponsa, Thierry, \& Duñabeitia, 2020; Hoversten, Brothers, Swaab, \& Traxler, 2017). Therefore, it seems plausible that sub-lexical factors such as orthotactic distinctiveness play a key role in determining the 
language of words during visual word recognition (see also Oganian, Conrad, Aryani, Heekeren, \& Spalek, 2016; Vaid \& Frenck-Mestre, 2002). And, in the absence of additional contextual cues, multilingual single word recognition is a process that initially requires a fast-acting language detection mechanism. Consequently, orthotactics should have a direct impact in second language learning through correct and efficient language categorization.

Preceding research on language categorization suggests differential development of sequential bilinguals' linguistic systems (Segalowitz, 1991; Van Kesteren et al., 2012); it assumes that the native language is stable through time while the second language is the one that changes the most throughout acquisition and consolidation. It is thus expected that the native language influences the second language, and not the other way around. Evidence in support of this assumption comes from studies showing that second language learners normally exhibit difficulties with L2 accent and prosody, with spillover or transfer effects from their L1. This evident L2 malleability has led some authors to characterize the native language as stable and resistant and the L2 weak as impressionable (Frenck-Mestre \& Pynte, 1997; Hernandez, Bates, \& Avila, 1994). However, and not surprisingly, recent evidence shows that not the L2 but also the L1 changes during learning (see, among many others, Baus, Costa, \& Carreiras, 2013; Kroll, Dussias, Bice, \& Perrotti, 2015).

While L2 language learning abilities can extend beyond young adulthood, the malleability of the native language as a function of the acquisition of a new language seems to diminish with increasing age (Macwhinney, 2007; Schmid \& Köpke, 2017). In spite of the cognitive decline associated with ageing (Harris et al., 2009), language learning can effectively take place late in life ( see Antoniou \& Wright, 2017; Ramos, Fernández García, Antón, Casaponsa, \& Duñabeitia, 2017; Ware et al., 2017). The question of interest here is whether $L 2$ acquisition late in life impacts L1 orthotactic structure. Thus, the present study focuses on older adults as a critical test group. It is worth noting that the sensitivity to violations of the orthotactic rules of the first and 
second language has already been shown in younger bilingual adults to certain extent (Oganian,

113 Conrad, Aryani, Spalek, \& Heekeren, 2015), suggesting that L2 learning might have an impact in

114 L1 orthotactics. However, it is unclear whether similar L1 changes can be observed in older populations, when presumably the resistance to change and stability of L1 is at its peak, and the malleability and plasticity of the language system is at its lowest.

Hence, the present longitudinal study aims to investigate whether older adults are sensitive to markedness before learning a second language, and how this learning process changes their sensitivity to orthographic regularities. Specifically, we tested whether language learning late in life and the progressive improvement in L2 skills modulated learners' sensitivity not only to L2 orthotactics, but also to the orthotactic structure of the L1. To this end, older native Spanish speaker adults immersed in a Basque language-learning course for two consecutive academic years were tested in three critical moments (before, during, and after language learning) on their sensitivity to orthotactics via a language discrimination task. We decided to use a twoalternative forced-choice language decision task on pseudowords to minimize the influence of pre-existing L1 lexical and semantic knowledge (see Oganian et al., 2016, for a similar procedure).

\section{Methods}

Participants

Thirty retired Spanish monolingual adults took part in this longitudinal experiment. However, only twenty participants remained through the two year sessions ( 8 females; mean age $=66.57 ; S D=5.56)$. All participants were living in the Basque Country, a Spanish region with two coexisting co-official languages, Spanish and Basque. None of the participants had prior knowledge of Basque, neither could they understand or produce linguistic structures in any 
other language than Spanish (see below). All participants reported having normal or correctedto-normal vision, and none of them had any history of chronic neuropsychological disorders.

Participants were recruited by advertisement at a Center of Continuing Education for Adults where free Basque lessons were offered to retired Spanish monolingual adults with no prior knowledge of Basque. This experiment was part of a larger project suported by the Basque Government to study the impact of second language acquisition in the elderly on other cognitive capacities, such as inhibitory control (Antón, Fernández García, Carreiras, \& Duñabeitia, 2016) and switching ability (Ramos et al., 2017). Participants signed a written consent form approved by the Ethics and Research Committees of the Basque Center on Cognition, Brain, and Language ( $B C B L)$ before the start of the research and educational actions.

Participants undertook Basque lessons for two whole academic years at the Center of Continuing Education for Adults. They attended Basque lessons for a period of eight months each year. Small groups of a maximum of 10 participants per class were arranged. In total, five hours and a half of training were set per week, distributed in three sessions held during working days. Participants were tested at the beginning of the academic year (T1), at the end of that same academic year (T2), and at the end of the second year of taking Basque lessons (T3). The linguistic project was coordinated by the Department of Education, Linguistic Policy and Culture of the Basque Government, and managed by native Basque-Spanish bilingual professional language trainers specialized in adult teaching.

At the beginning of the first academic year, all participants completed a general assessment consisting of a series of cognitive and language proficiency tasks. Age-related cognitive functioning was assessed using the Spanish version of the Mini-Mental State Examination (MMSE; see Lobo, Ezquerra, Gómez, Sala, \& Seva, 1979). Participants' linguistic profile before learning Basque was characterized via self-report measures of proficiency, and all participants were asked to rate their knowledge of Spanish and Basque on a scale from 1 to 10 
162 (see Table 1). Also, teachers evaluated participants' Basque proficiency based on their own

163 perception before the lessons started, ensuring that they did not have previous knowledge of

164 Basque. Self- and teacher-perceived Basque proficiency levels were also assessed at the end of

165 the learning process, together with additional objective measures of Basque knowledge. As

166 objective measures of language learning, participants completed a picture naming test (de Bruin,

167 Carreiras, \& Duñabeitia, 2017) in which participants had to name sixty-five common names in

168 Basque (see Table 1), and the beginner language test (A1 level) of the Common European

169 Framework for Reference (CEFR, Council of Europe, 2011), with a maximum score of 20.

170

171

Table 1. Descriptive statistics of the assessment

\begin{tabular}{lc}
\hline \hline Before Basque lessons & \\
\hline Age & $65.2(3.81)$ \\
Cognitive function (MMSE) & $28.8(1.24)$ \\
Self-perceived Spanish competence & $8.1(0.55)$ \\
Self-perceived Basque competence & 0 \\
Teacher-perceived Basque competence & 0 \\
\hline
\end{tabular}

\section{After Basque lessons}

Self-perceived Basque competence

$5.75(1.45)$

Teacher-perceived Basque competence

6.15 (1.09)

A1 level score

$19.7(4.28)$

Picture naming

$27.85(10.26)$

172

173

174

175

176

Note. Values reported correspond to the means (and standard deviations in parenthesis) of the age in years, result of the MMSE test, self-perceived Spanish and Basque skills (0-10 scale), teacher-perceived Basque competence (0-10 scale), score in the A1 level test (with a maximum score of 20 ), and number of correctly named pictures in a picture naming test.

\section{Materials and procedure}

First, a corpus of bigrams was constructed with the Spanish words from the B-PAL (Davis \& Perea, 2005) and Basque words from the E-HITZ (Perea et al., 2006) databases, and filtered with the items contained in the SYLLABARIUM database (Duñabeitia, Cholin, Corral, Perea, \& Carreiras, 2010). Words that contained letters that do not exist in the other language were removed (e.g., c, ñ, v). Bigrams that did not appear in any form in the other language were considered illegal and were selected for the construction of the marked pseudowords. Bigrams 

times in different words of each database. One hundred and thirty-five pseudowords were generated with the help of Wuggy (Keuleers \& Brysbaert, 2010), manipulating the presence or absence of distinctive bigrams of each language. Forty-five of these pseudowords were Spanishmarked items, forty-five were Basque-marked items, and forty-five were language-neutral pseudowords. Marked pseudowords were created making sure that at least one of the constituent bigrams violated the orthotactics of the other language. For instance, 'txamur' is considered a Basque-marked pseudoword because the bigram ' $t x^{\prime}$ ' does not exist in Spanish (namely, the ' $t x$ ' bigram has a frequency of use of 0 in Spanish). On the other hand, neutral pseudowords were created using bigrams that were plausible in both languages, such as the bigram 'rd' that exists in words such as cerdo (the Spanish word for pig), or ardi (the Basque word for sheep). Those neutral bigrams were controlled to have equal mean frequency of use in Spanish and Basque, $t(44)=0.03, p=.976$, Cohen's $d=.033$ (see Table 2). The position- and lengthdependent mean bigram frequency of each pseudoword as provided by B-PAL and E-Hitz databases was calculated the sets of pseudowords were matched based on this measure. This way, neutral pseudowords had an overall mean bigram frequency similar to that of Spanishmarked pseudowords when measured according to the Spanish statistics, and similar to that of Basque-marked pseudowords when measured according to the Basque statistics. This ensured that neutral pseudowords were equally legal in both languages when position-dependent and length-dependent measures were taken into account. Furthermore, the number of orthographic neighbours in Spanish and Basque were controlled to be similar for neutral pseudowords and for marked pseudowords (see Table 2). 
210 Table 2. Descriptive statistics of characteristics of the materials

\begin{tabular}{lccc}
\hline \hline & $\begin{array}{c}\text { Neutral } \\
\text { pseudowords }\end{array}$ & $\begin{array}{c}\text { Spanish-marked } \\
\text { pseudowords }\end{array}$ & $\begin{array}{c}\text { Basque-marked } \\
\text { pseudowords }\end{array}$ \\
\hline Length & $5.88(1,46)$ & $6.11(1,35)$ & $6.11(1,54)$ \\
Neighbors in Spanish & $1.75(2,49)$ & $0.35(1,19)$ & $1.91(2,19)$ \\
Neighbors in Basque & $1.6(2,15)$ & $1.64(2,67)$ & $0.2(0,69)$ \\
Mean bigram frequency in Basque & $2.06(0,35)$ & $0.68(0,29)$ & $2.10(0,29)$ \\
Mean bigram frequency in Spanish & $2.24(0,54)$ & $2.25(0,52)$ & $0.91(0,39)$ \\
Illegal bigram frequency in Basque & $0(0)$ & $1.31(0,51)$ & $0(0)$ \\
Illegal bigrams frequency in Spanish & $0(0)$ & $0(0)$ & $1.37(0,61)$ \\
\hline \hline
\end{tabular}

Note. Values reported are means and standard deviation in parenthesis on word length (number of letters), orthographic neighbours (number of words that share all letters but one), mean bigram frequency (position- and length-dependent mean bigram frequency as extracted by B-PAL and E-HITZ), and illegal bigrams (extracted from total counts of the LEXESP and SILLABARIUM databases).

217 by a trained research assistant who accompanied them during the course of the whole language learning process. The same computer was used at all test moments in order to avoid any hardware-related differences across sessions. The experiment was programmed in Experiment Builder (SR Research, Ontario). The start of each trial was marked by a fixation cross appearing in the middle of the screen for $500 \mathrm{~ms}$, immediately followed by the target word for $3000 \mathrm{~ms}$ or until participants' response. At the beginning of the task, participants performed some trials as practice. They were instructed to decide whether the string of letters appearing on the screen could belong to Spanish or Basque (i.e., forced-choice), and to do so as fast as possible. Participants were asked to press one out of two buttons in a handheld controller to indicate whether each string could belong to Spanish or Basque. Participants were informed that none of the strings appearing on the screen were real words. Participants were asked to perform this task before (T1) and after (T2) the first academic year, and one year later (T3). 
232 in the statistical environment R ( $R$ core team, 2013). Before data analysis, responses below 200 ms (0.01\%) and timeouts (0.04\%) were excluded. Also, responses that deviated 3.5 standard deviations above and below the mean from all within-subjects ( $1.05 \%$ of outliers) or withinitems $(0.43 \%$ of outliers) factors were excluded from the analyses, leading to a final rejection of $1.33 \%$ of the data. mixed-effects models (Baayen, Davidson, \& Bates, 2008; Barr, 2013; Jaeger, 2008), using Ime4 package for R (Bates, Mächler, Bolker, \& Walker, 2015). We first fitted maximal random structure models. When the data did not support the execution of the maximal model random structure, we then reduced the model complexity in order to arrive at a parsimonious model. To do so, we computed principal component analyses (PCA) of the random structure (see Bates et al., 2015), and then kept the number of principal components that cumulatively accounted for $100 \%$ of the variance. Type-III Anova Wald-tests was obtained to assess the significance of fixed effects for binary data, and Type-III Anova F-tests with Satterwhite approximations to degrees of freedom were obtained for response latency analysis. Averaged reaction times and accuracy rates per condition are presented in Table 3 . Considering that decisions made on neutral pseudowords cannot be characterized as correct or incorrect responses in the absence of language cues, response latencies for these items were modelled by the type of response. The response tendency was based on the given response of the participants, being dummy coded as ' 1 ' if participants responded Spanish and ' 0 ' if they responded Basque (see Table 3). In contrast, in the case of marked pseudowords, the percentage of correct responses was analyzed based on the presence of language cues, and reaction times were analysed using only correct answers (see Table 3).

First, we investigated whether Type of Markedness (Neutral, Spanish-marked, Basquemarked) and Test Moment ( $\mathrm{T} 1, \mathrm{~T} 2, \mathrm{~T} 3)$ had an overall impact on participants' language choice. 
257 Then, we analysed marked and neutral pseudowords separately, given the low proportion of

258 "other" language choices for marked conditions (i.e., Spanish-marked pseudowords and Basque-

259 marked pseudowords were correctly categorised as Spanish and Basque, respectively, more

260 than $90 \%$ of the cases; see Table 3). Note also that responses for neutral pseudowords cannot

261 be categorized as correct or incorrect responses for obvious reasons. Thus, response latencies

262 for neutral psuedowords were analysed including Test Moment (T1, T2, T3) and Response Type

263 (Spanish, Basque) as predictors. Reaction times and accuracy data of marked pseudowords was

264 analysed including Test Moment (T1, T2, T3) and Type of Markedness (Basque-marked, Spanish-

265 marked) as predictors.

266

Table 3. Descriptive statistics for the language decision task in the three different test moments (T1, T2 and T3). The values reported correspond to the means and standard deviations (in parenthesis) of the accuracy rates (\% of hits) and of the reaction times (in milliseconds).

\begin{tabular}{lcccc}
\hline \hline & \multicolumn{2}{c}{ Marked } & \multicolumn{2}{c}{ Neutral } \\
\hline ACCURACY & Basque & Spanish & Basque tendency & Spanish tendency \\
\hline T1 & $92.44(26.45)$ & $91.12(28.46)$ & $32.36(20.56)$ & $67.64(46.81)$ \\
T2 & $94.33(23.14)$ & $94.72(22.38)$ & $28.03(18.79)$ & $71.97(44.94)$ \\
T3 & $93.2(25.18)$ & $91.74(27.54)$ & $28.04(21.38)$ & $71.96(44.95)$ \\
\hline REACTION TIMES & Basque & Spanish & Basque tendency & Spanish tendency \\
\hline T1 & $873(296)$ & $1003(446)$ & $1295(559)$ & $1050(454)$ \\
T2 & $897(306)$ & $953(380)$ & $1269(526)$ & $1029(467)$ \\
T3 & $883(283)$ & $898(297)$ & $1276(519)$ & $1014(441)$ \\
\hline \hline
\end{tabular}

Results

Overall response choices

274 (Spanish-marked, Basque-marked, and Neutral) across Test Moments (T1, T2, and T3 ). Analyses revealed a main effect of language markedness $\left[\chi^{2}(2)=199.72, p<.001\right]$, such that the tendency of Spanish responses was higher for Spanish-marked pseudowords as compared to neutral

277 pseudowords [ $b=2.32, \mathrm{SE}=0.44, z=5.23, p<.001]$, and for neutral pseudowords as compared to 
Basque-marked pseudowords [ $b=5.25, \mathrm{SE}=0.42, z=12.55, p<.001]$. We did not find a significant main effect of the Test Moment $\left[\chi^{2}(2)=2.63, p=.275\right]$. The interaction between Type of Markedness and Test Moment was significant $\left[\chi^{2}(4)=13.03, p=.01\right]$. However, post-hoc analyses revealed no significant differences across Test Moment for neutral (all ps>.26), Basque-marked (all ps>.40), or Spanish-marked pseudowords (all ps>.21).

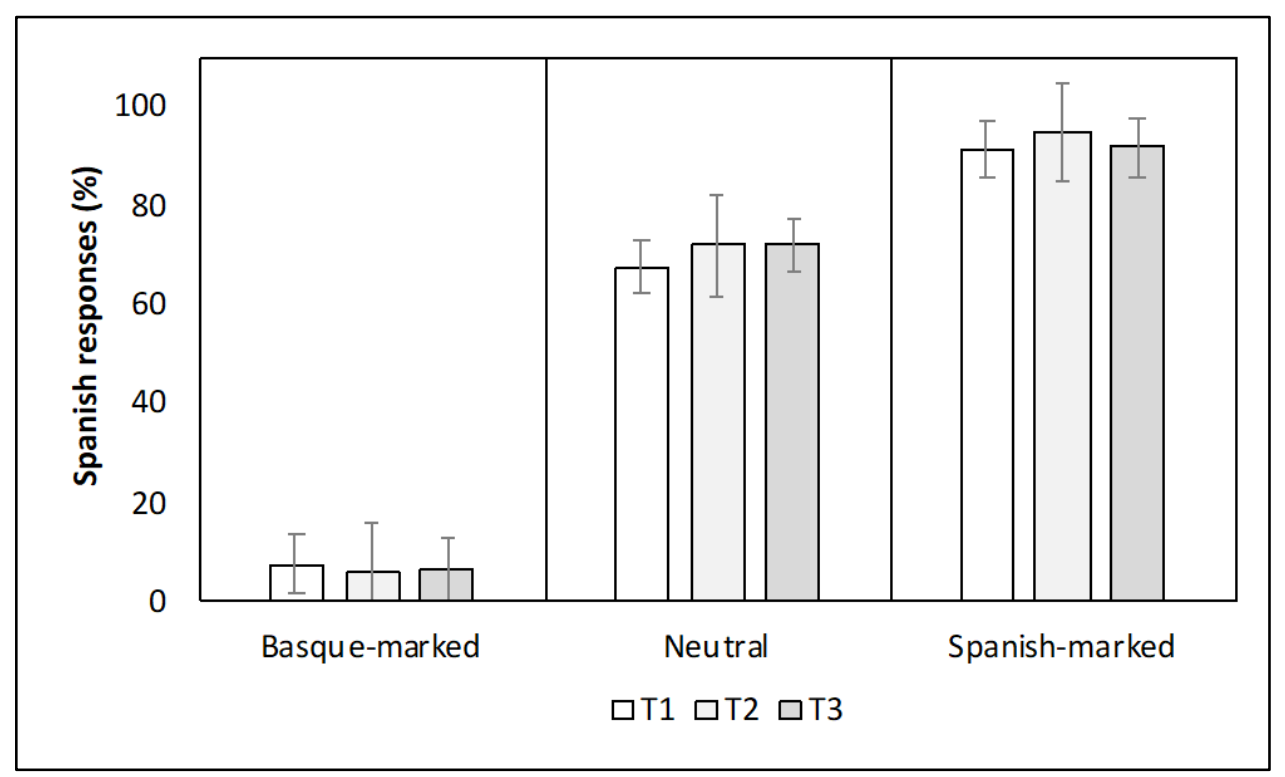

Figure 1. Percentage of Spanish responses to Basque-marked, neutral, and Spanish-marked pseudowords before language learning (T1), after one year of language learning (T2), and after two years (T3). Error bars represent \pm 1 standard error (SE) of the mean. interaction between Test Moment and Response $[F(2,2202.14)=2.07, p=.13]$ were not significant. 
The analysis of congruent language selection responses based on the presence of Type of Markedness (Spanish-marked, Basque-marked) across Test Moments did not reveal any significant main effect or interaction (all ps $>$.12). Overall accuracy ratings were already close to ceiling at T1 for both the Spanish-marked and Basque-marked items (see Table 3).

Analyses of reaction times on marked pseudowords revealed a main effect of Type of Markedness $[F(1,36.3)=6.46, p=0.02]$, indicating that participants were overall slower at detecting Spanish-marked than Basque-marked pseudowords [ $b=90.25, \mathrm{SE}=35.51$; see Figure 2]. The main effect of Test Moment was not significant $[F(2,19)=.83, p=.45]$. Importantly, a significant interaction was found between Type of Markedness and Test Moment $[F(2,4778)=20.89, p<.001]$. Planned comparisons revealed that whilst in T1 participants were significantly slower at responding to Spanish-marked pseudowords as compared to Basquemarked pseudowords [i.e., markedness effect; $b=155.73, \mathrm{SE}=36.97, \mathrm{t}(42.6)=4.21, p<.001$ ], this difference diminished after language learning $[T 2: b=67.81, S E=436.90, t(42.3)=1.84, p=.07 ; T 3$ : 47.21, $\mathrm{SE}=36.995, \mathrm{t}(42.7)=1.28, p=.21]$. This reduction of the markedness effect over the different test moments was due to an overall reduction in response latencies to Spanish-marked pseudowords [ $\mathrm{b}=96.31, \mathrm{SE}=41.19, \mathrm{t}(20.9)=2.34, p=.03$ ], whilst Basque-marked pseudoword response latencies remained constant $[\mathrm{b}=-12.21, \mathrm{SE}=41.13, \mathrm{t}(20.7)=-.30, p=.77]$. 


\section{Neutral}

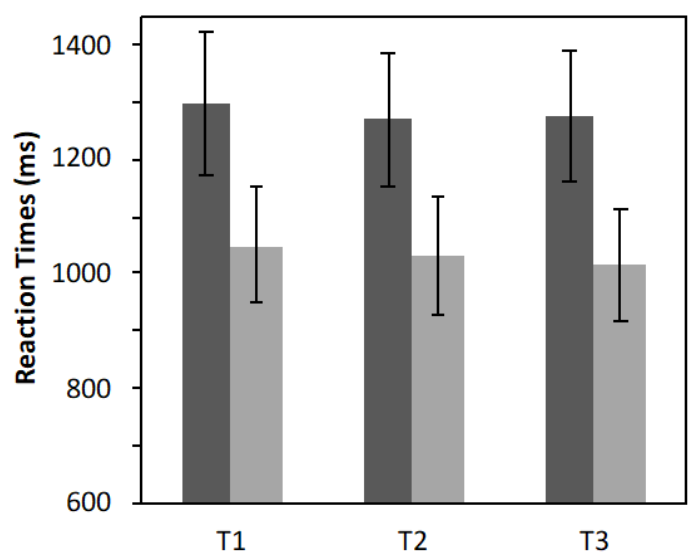

Marked

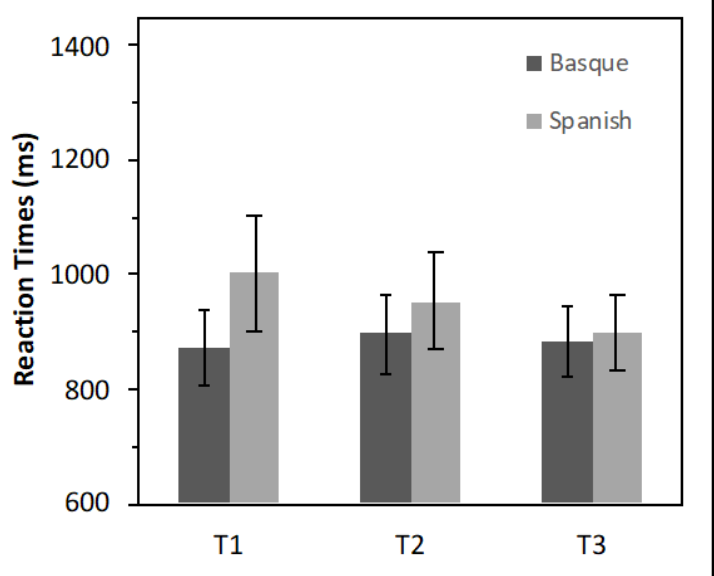

Figure 2. Bar plots depicting participants' response latencies in the language decision task for neutral (left) and marked (right) pseudowords in T1, T2, and T3. For neutral pseudowords, all Basque (dark grey) and Spanish (light grey) responses are included. For marked pseudowords, only responses congruent with the marked type are included. Error bars represent \pm 1 standard error (SE) of the mean.

\section{Discussion}

The present longitudinal study investigated changes in orthotactic sensitivity in a group of older Spanish monolingual adults before and after they learned Basque. Our results confirmed previous findings showing that older adults were highly sensitive to orthotactic markedness in Basque, as shown by their faster reaction times when responding to Basque-marked words (Casaponsa et al., 2014; Duñabeitia, Ivaz, \& Casaponsa, 2016; Oganian, Conrad, Aryani, Heekeren, \& Spalek, 2016). This sensitivity to L2 markedness was shown even before participants learned Basque, and it persisted during the learning process. However, and more importantly, we also found that participants demonstrated increased sensitivity to orthotactic markedness in their native language after learning a second language, evidenced by faster reaction times. This strongly suggests that sensitivity to native orthotactics changes due to the accommodation of newly acquired regularities from a second language.

As shown in the current study, before and after learning a second language, the presence of language-specific orthotactic cues guides and aids language classification. Our 
participants were able to easily classify Basque-marked and Spanish-marked pseudowords as

337 Basque and Spanish, respectively, depite their complete lack of Basque knowledge. However, when participants classified seemingly neutral pseudowords without language-specific orthotactic cues, they showed a strong preference to classify them as belonging to their native language, Spanish. This effect was also accompanied by faster reaction times for the neutral pseudowords which they deemed to be Spanish. One possible explanation for this finding is that readers consider familiar orthotatic patterns to be part of their previous knowledge. In line with this assumption, previous research (Ellis \& Beaton, 1993) has shown that people prefer to learn letter sequences that follow sequences found in their native language, suggesting they have a preference for patterns that follow or align with the $\mathrm{L} 1$ orthotactic rules.

In general terms, participants showed high sensitivity levels to orthographic markedness, responding significantly faster to marked than to neutral pseudowords in both languages, both before and during second language learning. Even though older adults were equally accurate at detecting Spanish-marked as they were at detecting Basque-marked pseudowords, they responsed more quickly to Basque-marked pseudowords. However, this was only true in the T1, when they had not yet learned Basque. This suggests that before learning the second language, participants could easily realize that Basque-marked pseudowords did not conform to the L1 orthotactic regularities. These results are in line with previous findings from the orthotactic standards set by the orthographic distributional properties of the native language is not a trivial one, other findings provide additional insights regarding the dynamic nature of the orthographic system. Interestingly, results from the two other test moments (T2 
361

362

363

364

365

366

367

368

369

370

371

372

373

374

375

376

378

379

380

381

382

383

384

385

and T3) suggest that the probabilistic distribution of regularities in the native language changes.

While accuracy in detecting the language of marked pseudowords remained very high and relatively constant across the three test moments, the analysis of reaction times showed significant variations depending on the type of pseudowords. Basque-marked pseudowords were detected equally fast across the three test moments, but reaction times to Spanish-marked pseudowords decreased significantly as a function of increased exposure to the new language.

It could be tentatively argued that this reduction in reaction times associated with Spanishmarked pseudowords could be associated with a change in the response strategy. In a first test moment, participants could have had carefully evaluated if the pseudowords belonged to Spanish by assessing their degree of similarities with known Spanish words, and then stop using this strategy once they became familiar with the task, resulting in faster reaction times across sessions. However, this may not seem to be a valid explanation that fits all the data, since if this were the case, participants would have shown faster responses over time for all types of pseudowords. We believe that similar automatic sub-lexical and lexical competition and selection mechanisms guided participants' responses in the three test sessions, as predicted by current bilingual interactive activation models.

Hence, the current pattern could be readily accounted for by bilingual word identification models that predict different processing mechanisms as a function of the sublexical characteristics of the items (i.e., see BIA+ extended, Van Kesteren et al., 2015; see also BIA+ S, Casaponsa et al., 2020). In the case of neutral words, responses were mainly influenced by the formal similarity with existing lexical entries from the native language lexicon, consequently leading to faster Spanish choices compared to Basque choices (see Figure 2). Not surprisingly, responses to neutral pseudowords were heavily influenced by the native language even after learning Basque, leading to similar choices and responses latencies across sessions. It should be noted in this regard that the general level of $\mathrm{L} 2$ achieved was admittedly low (namely, 
A1 level of CEFR), and accordingly the degree of L2 lexical consolidation was low too. In this line,

387 Casaponsa, Antón, Pérez and Duñabeitia (2015) showed that at A1 levels, the speed of response

to L2 words is indeed heavily influenced by L1 knowledge, coinciding with the findings of the current study. In the case of marked pseudowords, the mechanisms that underlie language identification differ for Spanish-like and Basque-like strings. On the one hand, responses to Basque-marked pseudowords were mainly driven by the earliest stages of orthographic processing, leading to faster reaction times as compared to Spanish-marked pseudowords. Importantly, these decisions were not affected by $L 2$ proficiency, leading to similar reaction times across sessions (see Casaponsa et al., 2014, for similar results; see also BIA+S, Casaponsa et al., 2020). On the other hand, responses to Spanish-marked pseudowords appeared to be less mediated by sub-lexical stages of processing and more mediated by lexical search routines at initial stages of language learning, resulting in significantly slower reaction times at T1. We suggest that the reliance on specific L1 and L2 orthotactic information progressively increased as participants learned Basque, and that the response criteria for Spanish-marked pseudowords shifted from a lexical search at T1 to a sub-lexical strategy at T2 and T3, allowing participants to speed up their language decision process for strings that violated L2 orthotactics. sub-lexical language nodes (see BIA+ extended, Van Kesteren et al., 2015; see also BIA+S, 404 Casaponsa et al., 2019). These accounts predict that the activation of the sub-lexical language 405 nodes due to the presence of language-specific sub-lexical cues will speed up language decision processes once the orthotactic rules of the first and the second language are integrated in the system. In the absence of sub-lexical language cues, the language decision process would be guided by lexical language nodes, and hence influenced by lexical competition and selection mechanisms. Thus, the current results fit well with these accounts, and they suggest that participants developed increased sensitivity to orthotactic regularities specific to their native 
411 language as a function of second language learning. This finding is particulary interesting as it

412 suggests that learning a second language changes the sensitivity to the orthotactics of the native

413 language (see also Casaponsa et al., 2014, suggesting that bilinguals' sensitivity to markedness

414 changes as a function of proficiency).

Learning a language implies, among many other things, integrating new words within

the set of existent representations of the native language. Therefore, while learning a second language, people also learn the similarities and differences between the to-be-incorporated words and the already known ones. The construction of the orthotactic repertoire is thus an automatic and spontaneous parallel process that takes place as a result of visual word processing. Learners need to implicitly acquire new orthotactic regularities and compare these with already known (native) patterns in order to make links between the new and the existing pieces of information. Thus, it seems plausible that as readers compare the new patterns with the old ones, they become more sensitive to the specificities of the old ones, consequently perceiving native orthotatic regularities differently. In other words, we propose that after learning a second language, readers may be better able to detect strings with native languagespecific cues due to increased saliency as pieces of orthotactic information that contrast with the newly acquired language. L1 remains static over time. Whilst the second language can be influenced by native language processing (Frenck-Mestre \& Pynte, 1997; Hernandez et al., 1994; Segalowitz, 1991), the native language itself has been typically considered as relatively impermeable and immutable. However, results in this study suggest that L1 orthotactic sensitivity changes while learning a second language. The idea that bilinguals' whole linguistic system displays adaptive changes was already proposed by Kroll, Dussias, Bice, and Perrotti (2015; see also Dussias and Sagarra, 2007). They hypothesized that the linguistic system is permeable in both languages, especially when 
high proficiency in L1 is achieved. The idea of native language changes pursuant to language

437 learning fits well with preceding studies suggesting that learning new words and grammar

438 interacts with the existing language in a dynamic way, changing the linguistic system as a whole 439 (Baus et al., 2013; Chang, 2013; Kartushina, Frauenfelder, \& Golestani, 2016; Linck, Kroll, \& 440 Sunderman, 2009). Following these premises, our results demonstrate that changes in the 441 linguistic system due to L2 learning can emerge even when the malleability of the native 442 language is presumably at its lowest. By means of testing older samples over a period of two years of language learning, we were able to show that lifelong exposure to a unique language system (namely, the native language), does not eliminate permeability to the properties of a new language. Furthermore, our results suggest that the sub-lexical mechanisms underpinning second language learning across the lifespan are relatively stable and qualitatively similar for old and young learners. Similar to young adults (see Oganian et al., 2016), older learners successfully rely on the acquisition of implicit knowledge when learning a second language, focusing on the statistical regularities of the sub-lexical units of their languages. permeable and changes during second language learning. Specifically, learning a new language that does not share native language orthotactics can change the perception of orthotactics in the native language already at early stages of $L 2$ acquisition. Future research should explore what other aspects of the native language may change as consequence of second language learning, and correctly characterize the stages and rythms at which these changes take place. This research will lead to a better understanding of the relationship between the native language and the multiligual linguistic system. 
460 Acknowledgements

461 This study was partially supported by grants RED2018-102615-T and PGC2018-097145-

462 B-I00 from the Spanish Government, H2019/HUM-5705 from the Comunidad de Madrid, and

463 by a fellowship from La Caixa foundation (ID 100010434, code LCF/BO/ES16/11570003). 
466

467

468

469

470

471

472

473

474

475

476

477

478

479

480

481

482

483

484

485

486

487

488

489

490

491

492

493

494

495

496

497

498

499

500

501

502

503

Antón, E., Fernández García, Y., Carreiras, M., \& Duñabeitia, J. A. (2016). Does bilingualism shape inhibitory control in the elderly? Journal of Memory and Language, 90, 147-160. https://doi.org/10.1016/j.jml.2016.04.007

Antoniou, M., \& Wright, S. M. (2017). Uncovering the mechanisms responsible for why language learning may promote healthy cognitive aging. Frontiers in Psychology, 8(DEC), 1-12. https://doi.org/10.3389/fpsyg.2017.02217

Baayen, R. H., Davidson, D. J., \& Bates, D. M. (2008). Mixed-effects modeling with crossed random effects for subjects and items. Journal of Memory and Language, 59(4), 390-412. https://doi.org/10.1016/j.jml.2007.12.005

Barr, D. J. (2013). Random effects structure for testing interactions in linear mixed-effects models. Frontiers in Psychology, 4(June), 3-4. https://doi.org/10.3389/fpsyg.2013.00328

Bates, D., Mächler, M., Bolker, B. M., \& Walker, S. C. (2015). Fitting linear mixed-effects models using Ime4. Journal of Statistical Software, 67(1). https://doi.org/10.18637/jss.v067.i01

Baus, C., Costa, A., \& Carreiras, M. (2013). On the effects of second language immersion on first language production. Acta Psychologica, 142(3), 402-409. https://doi.org/10.1016/j.actpsy.2013.01.010

Bordag, D., Kirschenbaum, A., Rogahn, M., \& Tschirner, E. (2017). The role of orthotactic probability in incidental and intentional vocabulary acquisition L1 and L2. Second Language Research, 33(2), 147-178. https://doi.org/10.1177/0267658316665879

Casaponsa, A., Antón, E., Pérez, A., \& Duñabeitia, J.A. (2015). Foreign language comprehension achievement: insights from the cognate facilitation effect. Frontiers in Psychology, 6:588.

Casaponsa, A., Carreiras, M., \& Duñabeitia, J. A. (2014). Discriminating languages in bilingual contexts: The impact of orthographic markedness. Frontiers in Psychology, 5(MAY), 1-10. https://doi.org/10.3389/fpsyg.2014.00424

Casaponsa, A., Carreiras, M., \& Duñabeitia, J. A. (2015). How do bilinguals identify the language of the words they read? Brain Research, 1624(August), 153-166. https://doi.org/10.1016/j.brainres.2015.07.035

Casaponsa, A., \& Duñabeitia, J. A. (2016). Lexical organization of language-ambiguous and language-specific words in bilinguals. Quarterly Journal of Experimental Psychology, 69(3), 589-604. https://doi.org/10.1080/17470218.2015.1064977

Casaponsa, A., Thierry, G., \& Duñabeitia, J. A. (2020). The role of orthotactics in language switching: An ERP investigation using masked language priming. Brain Sciences, 10(1), 22. https://doi.org/10.3390/brainsci10010022

Chang, C. B. (2013). A novelty effect in phonetic drift of the native language. Journal of Phonetics, 41(6), 520-533. https://doi.org/10.1016/j.wocn.2013.09.006

Chetail, F., \& Content, A. (2017). The perceptual structure of printed words: The case of silent $E$ words in French. Journal of Memory and Language, 97, 121-134. https://doi.org/10.1016/j.jml.2017.07.007 
Comesaña, M., Soares, A. P., Sánchez-Casas, R., \& Lima, C. (2012). Lexical and semantic representations in the acquisition of $L 2$ cognate and non-cognate words: Evidence from two learning methods in children. British Journal of Psychology, 103(3), 378-392. https://doi.org/10.1111/j.2044-8295.2011.02080.x

Conway, C. M., Bauernschmidt, A., Huang, S. S., \& Pisoni, D. B. (2010). Implicit statistical learning in language processing: Word predictability is the key. Cognition, 114(3), 356371. https://doi.org/10.1016/j.cognition.2009.10.009

Council of Europe, C. (2011). Common European framework of reference for languages: Learning, teaching, assessment. Cambridge, UK: Press Syndicate of the University of Cambridge.

Davis, C. J., \& Perea, M. (2005). BuscaPalabras: A program for deriving orthographic and phonological neighborhood statistics and other psycholinguistic indices in Spanish. Behavior Research Methods, 37(4), 665-671. https://doi.org/10.3758/BF03192738

de Bruin, A., Carreiras, M., \& Duñabeitia, J. A. (2017). The BEST dataset of language proficiency. Frontiers in Psychology, 8(MAR). https://doi.org/10.3389/fpsyg.2017.00522

Duñabeitia, J. A., Cholin, J., Corral, J., Perea, M., \& Carreiras, M. (2010). SYLLABARIUM: An online application for deriving complete statistics for Basque and Spanish orthographic syllables. Behavior Research Methods, 42(1), 118-125. https://doi.org/10.3758/BRM.42.1.118

Duñabeitia, J. A., Ivaz, L., \& Casaponsa, A. (2016). Journal of Cognitive Psychology Developmental changes associated with cross-language similarity in bilingual children. https://doi.org/10.1080/20445911.2015.1086773doi.org/10.1080/20445911.2015.10867 73

Dussias, P. E., \& Sagarra, N. (2007). The effect of exposure on syntactic parsing in Spanish English bilinguals. Bilingualism, 10(1), 101-116. https://doi.org/10.1017/S1366728906002847

Ellis, N. C., \& Beaton, A. (1993). Psycholinguistic Determinants of Foreign Language Vocabulary Learning. Language Learning, 43(4), 559-617. https://doi.org/10.1111/j.14671770.1993.tb00627.x

Frenck-Mestre, C., \& Pynte, J. (1997). Syntactic ambiguity resolution while reading in second and native languages. The Quarterly Journal of Experimental Psychology, 50A(1), 119148.

Harris, S. E., Houlihan, L. M., Corley, J., Gow, A. J., Starr, J. M., Penke, L., ... Rafnsson, S. B. (2009). Age-associated cognitive decline. British Medical Bulletin, 92(1), 135-152. https://doi.org/10.1093/bmb/ldp033

Hernandez, A. E., Bates, E. A., \& Avila, L. X. (1994). On-line sentence interpretation in SpanishEnglish bilinguals: What does it mean to be "in between"? Applied Psycholinguistics, 15(4), 417-446. https://doi.org/10.1017/s014271640000686x

Hoversten, L. J., Brothers, T., Swaab, T. Y., \& Traxler, M. J. (2017). Early processing of orthographic language membership information in bilingual visual word recognition: Evidence from ERPs. Neuropsychologia, 103, 183-190. https://doi.org/10.1002/cncr.27633.Percutaneous 
Jaeger, T. F. (2008). Categorical data analysis: Away fron ANOVAs. Journal of Memory and Language, 59(4), 434-446. https://doi.org/10.1016/j.jml.2007.11.007.Categorical

Kartushina, N., Frauenfelder, U. H., \& Golestani, N. (2016). How and When Does the Second Language Influence the Production of Native Speech Sounds: A Literature Review. Language Learning, 66, 155-186. https://doi.org/10.1111/lang.12187

Keuleers, E., \& Brysbaert, M. (2010). Wuggy: A multilingual pseudoword generator. Behavior Research Methods, 42(3), 627-633. https://doi.org/10.3758/BRM.42.3.627

Krogh, L., Vlach, H. A., \& Johnson, S. P. (2013). Statistical learning across development: Flexible yet constrained. Frontiers in Psychology, 3(JAN), 1-11. https://doi.org/10.3389/fpsyg.2012.00598

Kroll, J. F., Dussias, P. E., Bice, K., \& Perrotti, L. (2015). Bilingualism, Mind , and Brain. Annual Review Linguistics, 1(1), 377-394. https://doi.org/10.1146/annurev-linguist-030514124937

Linck, J. A., Kroll, J. F., \& Sunderman, G. (2009). Linck, J. A., Kroll, J. F., \& Sunderman, G. (2009). Losing access to the native language while immersed in a second language: Evidence for the role of inhibition in second-language learning. Psychological Science, 20(12), 15071515. https://doi.org/10.1038/mp.2011.182.doi

Lobo, A., Ezquerra, J., Gómez, F. B., Sala, J. M., \& Seva, A. D. (1979). ognocitive mini-test (a simple practical test to detect intellectual changes in medical patients). Actas LusoEspanolas de Neurologia, Psiquiatria y Ciencias Afines, 7(3), 189-202.

Macwhinney, B. (2007). A Unified Model A Unified Model. Social Sciences.

Miller, G. A., Bruner, J. S., \& Postman, L. (1954). Familiarityofletter sequencesandtachistoscopicidentification. The Journal of General Psychology, 50(1), 129139.

Oganian, Y., Conrad, M., Aryani, A., Spalek, K., \& Heekeren, H. R. (2015). Activation patterns throughout the word processing network of L1-dominant bilinguals reflect language similarity and language decisions. Journal of Cognitive Neuroscience, 27(11), 2197-2214.

Oganian, Yulia, Conrad, M., Aryani, A., Heekeren, H. R., \& Spalek, K. (2016). Interplay of bigram frequency and orthographic neighborhood statistics in language membership decision. Bilingualism, 19(3), 578-596. https://doi.org/10.1017/S1366728915000292

Owsowitz, S. E. (1963). TheEffectsofWordFamiliarityandLetterStructure FamiliarityonthePerceptionofWords. SantaMonica,CA: RandCorporation Publications.

Perea, M., Urkia, M., Davis, C. J., Agirre, A., Laseka, E., \& Carreiras, M. (2006). E-Hitz: A word frequency list and a program for deriving psycholinguistic statistics in an agglutinative language (Basque). Behavior Research Methods, 38(4), 610-615. https://doi.org/10.3758/BF03193893

Ramos, S., Fernández García, Y., Antón, E., Casaponsa, A., \& Duñabeitia, J. A. (2017). Does learning a language in the elderly enhance switching ability? Journal of Neurolinguistics, 43, 39-48. https://doi.org/10.1016/j.jneuroling.2016.09.001

Schmid, M. S., \& Köpke, B. (2017). The relevance of first language attrition to theories of bilingual development. Linguistic Approaches to Bilingualism, 7(6), 637-667. 
Segalowitz, N. (1991). Does Advanced Skill in a Second Language Reduce Automaticity in the First Language? Language Learning, 41(1), 59-83. https://doi.org/10.1111/j.14671770.1991.tb00676.x

Vaid, J., \& Frenck-Mestre, C. (2002). Do orthographic cues aid language recognition? A laterality study with French-English bilinguals. Brain and Language, 82(1), 47-53. https://doi.org/10.1016/S0093-934X(02)00008-1

Van Kesteren, R., Dijkstra, T., \& de Smedt, K. (2012). Markedness effects in Norwegian-English bilinguals: Task-dependent use of language-specific letters and bigrams. The Quarterly Journal of Experimental Psychology, 65(11), 2129-2154.

Ware, C., Damnee, S., Djabelkhir, L., Cristancho, V., Wu, Y. H., Benovici, J., ... Rigaud, A. S. (2017). Maintaining cognitive functioning in healthy seniors with a technology-based foreign language program: A pilot feasibility study. Frontiers in Aging Neuroscience, 9(MAR), 1-10. https://doi.org/10.3389/fnagi.2017.00042 